\title{
Atrial fibrillation: relation between clinical risk factors and transoesophageal echocardiographic risk factors for thromboembolism
}

\author{
S Illien, S Maroto-Järvinen, G von der Recke, C Hammerstingl, H Schmidt, \\ S Kuntz-Hehner, B Lüderitz, H Omran
}

Heart 2003;89:165-168

See end of article for authors' affiliations

\section{Correspondence to}

Dr H Omran, Department

of Medicine-Cardiology,

University of Bonn,

Sigmund-Freud-Strasse 25

D-53105 Bonn, Germany;

omran@uni-bonn.de

Accepted

2 October 2002

\begin{abstract}
Objective: To correlate clinical risk factors for thromboembolism with transoesophageal echocardiography (TOE) markers of a thrombogenic milieu.

Design: Clinical risk factors for thromboembolism and TOE markers of a thrombogenic milieu were assessed in consecutive patients with non-rheumatic atrial fibrillation. The following TOE parameters were assessed: presence of spontaneous echo contrast, thrombi, and left atrial appendage blood flow velocities. A history of hypertension, diabetes mellitus, or thromboembolic events, patient age > 65 years, and chronic heart failure were considered to be clinical risk factors for thromboembolism. Setting: Tertiary cardiac care centre.

Patients: 301 consecutive patients with non-rheumatic atrial fibrillation scheduled for TOE.

Results: 255 patients presented with clinical risk factors. 158 patients had reduced left atrial blood flow velocities, dense spontaneous echo contrast, or both. Logistic regression analysis showed that a reduced left ventricular ejection fraction and age $>65$ years were the only independent predictors of a thrombogenic milieu (both $p<0.0001$ ). The probability of having a thrombogenic milieu increased with the number of clinical risk factors present $(p<0.0001) .17 .4 \%$ of the patients without clinical risk factors had a thrombogenic milieu whereas $41.2 \%$ of the patients presenting one or more clinical risk factors had none.

Conclusion: There is a close relation between clinical risk factors and TOE markers of a thrombogenic milieu. In addition, TOE examination allows for the identification of patients with a thrombogenic milieu without clinical risk factors.
\end{abstract}

$\mathrm{N}$ on-rheumatic atrial fibrillation (AF) is a common arrhythmia with a high prevalence in the elderly population. ${ }^{12}$ It is well known that $\mathrm{AF}$ is associated with an increased risk of ischaemic stroke. Previous studies reported event rates between $2-18 \%$ per year depending on the investigated patient population. ${ }^{3}$ Some studies have shown that the presence of clinical risk factors-arterial hypertension, diabetes mellitus, increased age, and depressed left ventricular ejection fraction (LVEF) - allows for the assessment of the thromboembolic risk in patients with $\mathrm{AF}^{4-7}$ Moreover, it has been shown that chronic oral anticoagulation decreases the risk of embolism in the latter patient groups. ${ }^{8-11}$ Nevertheless, thromboembolism may occur in patients without clinical risk factors. Furthermore, the individual risk of embolism may vary with different settings of clinical risk factors. ${ }^{45}$ The SPAF III (stroke prevention in atrial fibrillation) study showed that women older than 75 years, all patients older than 75 with a history of arterial hypertension, and patients with a blood pressure $>160 \mathrm{~mm} \mathrm{Hg}$ at the time of admission, have a particularly high risk for cerebral embolism. ${ }^{4}$ Similarly, the Atrial Fibrillation Investigators (AFI) found that patients older than 75 years and the presence of clinical risk factors indicate a substantially increased thromboembolic risk. ${ }^{5}$ More recent studies have shown that the risk of embolism in AF may also be determined by the use of transoesophageal echocardiography (TOE) markers of left atrial flow and function. Left atrial spontaneous echo contrast (SEC), a phenomenon of slow flow in the left atrium, has been correlated to increased risk of thrombus formation and thromboembolism. In addition, depressed left atrial appendage (LAA) function indicates an increased thromboembolic risk. ${ }^{32-16}$ Although those parameters are strong predictors of embolism in patients with $\mathrm{AF}$, their relation to clinical risk factors is not well established. Furthermore, it is not known whether assessment of TOE risk factors adds to clinical risk stratification in patients with AF. The aim of this study was to correlate clinical and transthoracic echocardiography (TTE) risk factors of thromboembolism with TOE findings and to evaluate whether TOE adds new information to clinical risk stratification.

\section{METHODS}

\section{Patients}

The study group consisted of consecutively recruited adult patients who were admitted to the department of cardiology at the University of Bonn with AF. Indications for TOE investigation were previous history of embolism (stroke, transient ischaemic attack, peripheral arterial embolism) and risk assessment before electrical cardioversion or implantation of an internal cardioverter/defibrillator device. The arrhythmia was diagnosed by standard 12 lead ECG and Holter monitoring. Patients with rheumatic heart valve disease or prosthetic heart valves were excluded from the study. A careful medical history was taken in all patients with special respect to their clinical risk factor profile, previous anticoagulation, and recent

Abbreviations: AF, atrial fibrillation; AFI, Atrial Fibrillation Investigators LAA, left atrial appendage, LVEF, left ventricular ejection fraction; SEC, spontaneous echo contrast; SPAF, stroke prevention in atrial fibrillation, TOE, transoesophageal echocardiography; TTE transthoracic echocardiography 
embolic events. All subjects underwent detailed clinical examination to assess underlying cardiovascular disease and the cause for AF.

\section{Clinical and TTE risk factors of thromboembolism}

According to the SPAF and AFI the following parameters were considered to be clinical risk factors of thromboembolic events $^{4-6}$ : a history of arterial hypertension with systolic blood pressures $>160 \mathrm{~mm} \mathrm{Hg}$ or diastolic blood pressures $>90 \mathrm{~mm} \mathrm{Hg}$ during repeated examinations or chronic antihypertensive treatment; diabetes mellitus; a history of thromboembolism (stroke, transient ischaemic attack, peripheral vascular embolism); and chronic heart failure with a reduced LVEF $<45 \%$ as assessed by standard TTE. Age $>65$ years was also considered a risk factor according to the results of the SPAF trials. ${ }^{4-6}$

\section{Echocardiography}

\section{Echocardiographic equipment}

The echocardiographic examinations were performed with commercially available equipment. We used a System $\mathrm{V}$ and a Vivid V from GE Ultrasound (G E Medical Systems, Wisconsin, USA) with a $1.7 / 3.4 \mathrm{MHz}$ harmonic transducer and a multiplane $6.7 \mathrm{MHz}$ transoesophageal probe. Measurements were performed off line with the Echopac software provided by the manufacturer.

\section{Echocardiographic studies}

All patients subsequently underwent TTE and TOE.

A standardised TTE examination including the standard imaging planes was performed according to the recommendations of the American Society of Echocardiography. ${ }^{17}$ Left ventricular volumes were assessed in the apical long axis and LVEF was calculated by using the Simpson method. The left atrial diameter was measured in the $M$ mode. Evidence for rheumatic heart valve disease led to exclusion from the study.

TOE was performed as previously described. ${ }^{18-20}$ Written consent was obtained from all patients. Patients were studied in the fasting state beginning four hours before examination. The oropharynx was anaesthetised by topical application of lidocaine spray. When needed light sedation with $2.5-5.0 \mathrm{mg}$ midazolam was given intravenously. After introduction of the multiplane transducer probe and adjustment of gain and compression settings a standardised examination was performed. The left atrium and the LAA were closely inspected for the presence of SEC and thrombi. SEC was classified in three degrees of intensity (none, mild SEC, dense SEC) as proposed by the SPAF investigators. ${ }^{15}$ The sample volume of the pulsed Doppler was placed $1 \mathrm{~cm}$ deep into the orifice of the LAA. A thrombogenic milieu was defined as the presence of dense SEC, LAA flow velocity $\leqslant 0.25 \mathrm{~m} / \mathrm{s}$, or both. Selected cine loops and Doppler velocity spectra were transferred to an external computer for subsequent analysis.

\section{Statistical analysis}

Data are presented as mean (SD). Nominal data were analysed with the $\chi^{2}$ test. The relation between clinical risk factors and a thrombogenic milieu was determined by multivariate logistic regression analysis. A probability value of $p \leqslant 0.05$ was considered to be significant. Furthermore, risk factor profiles defining patient subgroups at high risk of thromboembolism were correlated to the presence of a thrombogenic milieu. Therefore, all patients were subgrouped according to the SPAF III and AFI classification. ${ }^{45}$ The AFI analysed patients aged 65-75 years and patients aged $>75$ years separately. We pooled both groups because of their relatively small sizes. Data were analysed with the Statview version 5.0 software (Abacus, Berkeley, California, USA).
Table 1 Results of the multivariate logistic regression model showing the relation between clinical risk factors and the presence of a thrombogenic milieu

\begin{tabular}{lrcl}
\hline & $\mathrm{n}$ & $\mathrm{p}$ Value & $95 \% \mathrm{Cl}$ \\
\hline LVEF $<45 \%$ & 99 & $<0.0001$ & 1.84 to 5.67 \\
Age $>65$ years & 134 & $<0.0001$ & 2.6 to 7.57 \\
Arterial hypertension & 170 & 0.53 & 0.7 to 2.0 \\
Diabetes mellitus & 54 & 0.23 & 0.76 to 3.17 \\
Previous embolism & 51 & 0.64 & 0.59 to 2.39 \\
\hline
\end{tabular}

$\mathrm{Cl}$, confidence interval; LVEF, left ventricular ejection fraction.

\section{RESULTS}

\section{Patients}

Three hundred and one consecutive patients were included in the study. There were 229 men and 72 women. The mean age was 61 (11) years. Two hundred and thirty six $(78.4 \%)$ patients had permanent AF and 65 (21.6\%) patients had paroxysmal AF. The clinically estimated duration of the last episode was 26 (14) days. All patients were in AF at the time of the TOE assessment.

Prevalence of clinical risk factors of thromboembolism One hundred and seventy $(56.5 \%)$ patients had a history of arterial hypertension and 54 (17.9\%) had diabetes mellitus. A history of previous embolism was reported by 51 (16.9\%) patients. One hundred and thirty four $(44.5 \%)$ patients were older than 65 . Two hundred and fifty five $(84.7 \%)$ patients accumulated one or more of these risk factors (table 1).

\section{Echocardiographic studies}

All patients underwent TOE without complications. Imaging of the left atrium and LAA were feasible in all patients. LVEF was $<45 \%$ in $99(32.9 \%)$ patients as assessed by conventional TTE. The mean left atrial diameter was $4.7(0.8) \mathrm{cm}$.

SEC was visualised in $201(66.8 \%)$ patients. Mild SEC was present in $90(29.9 \%)$ patients and $111(36.8 \%)$ patients had dense SEC. Thrombi were found in 50 patients (16.6\%). All thrombi were situated in the LAA. Thirty nine thrombi adhered to the LAA wall and 11 were mobile. The thrombi measured $23(12) \times 15$ (7) $\mathrm{mm}$ (length $\times$ width). The mean LAA velocities was $0.34(0.23) \mathrm{m} / \mathrm{s}$. In $135(44.9 \%)$ patients LAA flow velocities were $\leqslant 0.25 \mathrm{~m} / \mathrm{s}$. One hundred and fifty eight $(52.5 \%)$ patients had dense SEC, LAA flow velocities $\leqslant 0.25 \mathrm{~m} / \mathrm{s}$, or both, thereby fulfilled the criteria of a thrombogenic milieu.

\section{Relation between a thrombogenic milieu and the presence of clinical risk factors}

Of patients with clinical risk factors, 150 (58.8\%) had a thrombogenic milieu. The remaining 105 patients (41.2\%) did not display a thrombogenic milieu. On the other hand eight patients $(17.4 \%)$ without clinical risk factors had a thrombogenic milieu. Univariate analysis showed that a history of arterial hypertension $(\mathrm{p}=0.049)$, diabetes mellitus $(p=0.0045)$, and age $>65$ years $(p<0.0001)$ were significantly correlated to the TOE finding of a thrombogenic milieu. A reduced LVEF of $<45 \%$ was also highly correlated with this finding $(\mathrm{p}<0.0001)$. However, a history of previous embolism showed no relation to the presence of a thrombogenic milieu $(p=0.11)$. The logistic regression model showed that a reduced LVEF of $<45 \%(\mathrm{p}<0.0001)$ and age $>65$ years $(p<0.0001)$ were the only independent predictors for the presence of a thrombogenic milieu. However, there was also a significant relation between higher age and the prevalence of diabetes mellitus $(\mathrm{p}=0.0014)$ and arterial hypertension $(\mathrm{p}=0.0049)$.

The statistical analysis showed a strong relation between the number of risk factors accumulated in one patient and the 


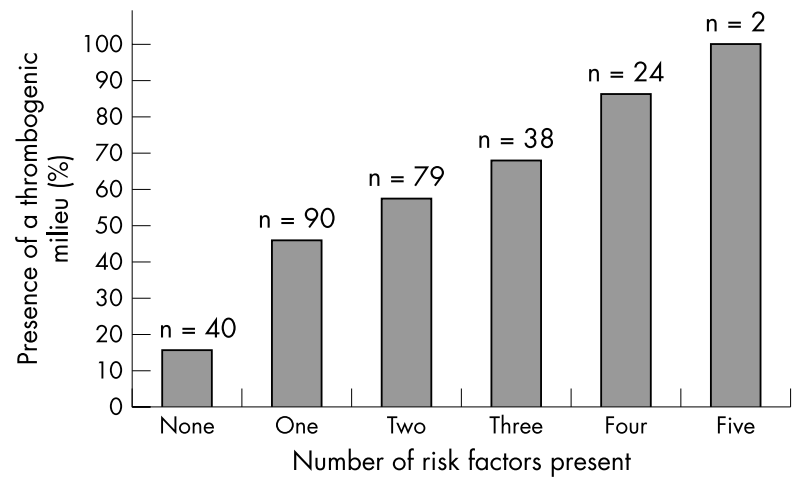

Figure 1 Relation between the number of clinical risk factors and the presence of a thrombogenic milieu.

prevalence of a thrombogenic milieu $(\mathrm{p}<0.0001)$. Figure $\mathrm{l}$ presents the results.

\section{Relation between the SPAF and AFI risk classification and the presence of a thrombogenic milieu}

Clinical identification of either high $(p=0.025)$ or moderate risk of thromboembolism ( $p=0.036)$ according to the SPAF III classification was significantly associated with the finding of a thrombogenic milieu as assessed by TOE. Similarly defining risk categories by the AFI criteria was significantly correlated to a thrombogenic milieu as assessed by TOE. Age $>65$ years in combination with other clinical risk factors was highly significantly related to the presence of a thrombogenic milieu $(p<0.0001)$. Furthermore, age $>65$ years in the absence of further risk factors $(p=0.04)$ was also correlated to a thrombogenic milieu. Table 2 presents the results.

\section{DISCUSSION}

In the past a lot of interest has been taken in identifying patients with AF who are at an increased risk of thromboembolism. Clinical risk factor have been well established. In addition, to assess the individual risk of thromboembolism clinically, TOE enables collection of further evidence of an increased thromboembolic risk. Previous studies showed that the presence of SEC-a phenomenon of slow flow in the atria-and depressed mechanical function of the LAA predispose to atrial thrombosis and are associated with an increased risk of thromboembolism. ${ }^{12}$ Leung and colleagues ${ }^{13}$ observed an annual thromboembolic event rate of $12 \%$ in patients with SEC compared with only $3 \%$ in patients without SEC. The SPAF III investigators found a relative risk of 2.7 for thromboembolism in patients with dense SEC..$^{14}$ Moreover, they observed that SEC was more frequent in the high risk patient group. However, it is not clear whether TOE risk assessment of thromboembolism adds to the clinical assessment of embolic risk in patients with AF. Furthermore, the incidence of a thrombogenic milieu in patients without clinical risk factors is unknown.

\section{Relation between clinical and TOE risk factors for thromboembolism}

Our data show that a history of hypertension, a history of diabetes mellitus, and age $>65$ years were all significantly related to the TOE finding of a thrombogenic milieu. In addition, LVEF $<45 \%$ was strongly associated with a thrombogenic milieu. The last finding is consistent with the results of previous TOE studies, which found that chronic heart failure and structural heart disease are predisposing factors for thromboembolism. ${ }^{21} 22$ Therefore, our data support the current guidelines for anticoagulation stating that older patients and patients with other clinical risk factors have an increased risk of thromboembolism. ${ }^{23}$ Interestingly, in our study a history of previous embolism was not significantly related to a thrombogenic milieu, although this parameter was shown in earlier anticoagulation trials to be an independent predictor for recurrent thromboembolism..$^{4-7}$ However, the rate of prior embolic events was relatively low in our patient population.

Another finding of our study was that multivariate analysis of risk factors showed that only age $>65$ years and LVEF $<45 \%$ were independent predictors of a thrombogenic milieu. This finding supports the clinical risk stratification, with older patients and patients with heart failure having a higher risk of thromboembolism. ${ }^{45} 23$

Based on the data of the large anticoagulation trials patient groups of high or low risk for thromboembolic events have been defined (table 2). ${ }^{4}$ Our data confirm this risk stratification showing that a high risk identification according to either the SPAF III or the AFI criteria were strongly correlated to a thrombogenic milieu. Furthermore, moderate risk as defined by SPAF III criteria were also significantly associated with a thrombogenic milieu. The AFI classified all patients in age dependent risk categories with and without additional clinical risk factors (table 2). Their analysis of pooled data from five anticoagulation trials found an increasing thromboembolic event rate with higher patient age and in the presence of clinical risk factors. Our data show that older patients more often have a thrombogenic milieu than younger patients, confirming this risk stratification.

Another interesting finding of this study was that the number of risk factors in individual patients was significantly correlated to the probability of having a thrombogenic milieu. The presence of a single clinical risk factor was associated with a $46 \%$ incidence of a thrombogenic milieu. Furthermore, the number of risk factors was closely correlated to the presence of a thrombogenic milieu. Thus, our data imply that patients with one or more of the above mentioned clinical risk factors should be considered for chronic oral anticoagulation.

Table 2 Risk categories according to the SPAF III and AFI data and their relation to the presence of a thrombogenic milieu

\begin{tabular}{llrc}
\hline Category & Classification criteria & $\mathrm{n}$ & $\mathrm{p}$ Value \\
\hline SPAF III, high risk & $\begin{array}{l}\text { Women }>75 \text { years or age }>75 \text { years with arterial } \\
\text { hypertension or systolic blood pressure }>160 \mathrm{~mm} \mathrm{Hg} \text { at } \\
\text { the time of admission }\end{array}$ & 22 & 0.025 \\
SPAF III, moderate risk & $\begin{array}{l}\text { Age } \leqslant 75 \text { years and a history of hypertension or a } \\
\text { history of diabetes mellitus }\end{array}$ & 173 & 0.036 \\
AFI & Age $<65$ years, no clinical risk factors & 46 & 0.29 \\
AFI & Age $<65$ years + clinical risk factors & 121 & 0.014 \\
AFI & Age $\geqslant 65$ years*, no clinical risk factors & 20 & 0.04 \\
AFI & Age $\geqslant 65$ years* + clinical risk factors & 114 & $<0.0001$ \\
\hline
\end{tabular}

*The AFI analysed patients aged 65 to 75 years and patients $>75$ years separately. $\mathrm{AFI}$, atrial fibrillation investigators; SPAF, stroke prevention in atrial fibrillation. 
The most interesting finding of our study was that a group of patients presenting with $\mathrm{AF}$ without any verifiable clinical risk factor had either dense SEC or poor LAA flow velocities. Thus, there seems to be a certain subgroup of patients with an increased risk of thromboembolism who would be missed by clinical risk stratification. In light of the high probability of thromboembolism in patients with a thrombogenic milieu anticoagulation should be considered in these patients.

\section{Study limitations}

The number of patients in this study was relatively small. In future, larger trials including the collection of follow up data on embolic event rates will have to evaluate the cost effectiveness of routine TOE screening in patients with AF, as well as its contribution to decreasing the embolic event rate.

\section{Conclusion and clinical implications}

The TOE finding of a thrombogenic milieu and the presence of clinical risk factors are closely correlated. Thus, the results of the present study confirm the value of the clinical risk stratification proposed by the SPAF III trial and the results of the AFI group. The strongest predictors of a thrombogenic milieu as assessed by TOE are a reduced LVEF of $<45 \%$ and age $>65$ years. Interestingly, there was a subgroup of patients presenting a thrombogenic milieu in the absence of any clinical risk factors. Thus, TOE may help to identify patients at risk for thromboembolism despite negative clinical risk factors. TOE risk stratification may be of particular value in patients where oral anticoagulation my be counterindicated or in younger patients without clinical risk factors.

\section{Authors' affiliations}

S Illien, S Maroto-Järvinen, G von der Recke, C Hammerstingl, H Schmidt, S Kuntz-Hehner, B Lüderitz, H Omran, Department of Medicine-Cardiology, University of Bonn, Bonn, Germany

\section{REFERENCES}

1 Wolf PA, Abbot RD, Kannel, WB. Atrial fibrillation as an independen risk factor for stroke: The Framingham study. Stroke 1991;22:983-8.

2 Furberg CD, Psaty BM, Manolino TA, et al. Prevalence of atria fibrillation in elderly subjects (the cardiovascular health study). Am J Cardiol 1994;74:238-41.

3 Kamp O, Verhorst PM, Welling RC, et al. Importance of left atrial appendage flow as a predictor of thromboembolic events in patients with atrial fibrillation. Eur Heart J 1999;20:979-85

4 Atrial Fibrillation Investigators. Risk factors for stroke and efficacy of anti thrombotic therapy in atrial fibrillation: Analysis of pooled data from five randomized trials. Arch Intern Med 1994;154:1449-57.
5 Hart RG, Pearce LA, McBride R, et al. Factors associated with ischaemic stroke during aspirin therapy in atrial fibrillation. Analysis of 2012 participants in the SPAF I - III clinical trials. Stroke 1999;30:1223-9.

6 Stroke Prevention in Atrial Fibrillation Investigators. Predictors of thromboembolism in atrial fibrillation. I. Clinical features of patients at risk. Ann Intern Med 1992;116:1-5.

7 Kalra L, Perez I, Melbourn A. Risk assessment and anticoagulation for primary stroke prevention in atrial fibrillation. Stroke 1999;30:1218-22.

8 Petersen P, Boysen G, Godtfredsen J, et al. Placebo controlled, randomized trial of warfarin and aspirin for prevention of thromboembolic complications in chronic atrial fibrillation; The Copenhagen AFASAK study. Lancet 1989;i:175-9.

9 EAFT Study Group. Secondary prevention in nonrheumatic atria fibrillation after transient ischaemic attack or minor stroke: EAFT (European atrial fibrillation trial) study group. Lancet 1993;342:125562.

10 Stroke Prevention in Atrial Fibrillation Investigators. Adjusted dose warfarin versus low-intensity, fixed dose warfarin plus aspirin for high risk patients with atrial fibrillation; Stroke prevention in atrial fibrillation III randomized clinical trial. Lancet 1996:348:633-8.

11 Hart RG, Benavente O, McBride R, I. Antithrombotic therapy to prevent stroke in patients with atrial fibrillation. A metanalysis. Ann Intern Med 1999;131:492-501.

12 Fatkin D, Kelly RP, Fenely MP. Relations between left atrial appendage blood flow velocity, spontaneous echocardiographic contrast and thromboembolic risk in vivo. J Am Coll Cardiol 1994:23:961-9.

13 Leung DYC, Black IW, Cranney GB, et al. Prognostic implications of left atrial appendage echo contrast in nonvalvular atrial fibrillation. J Am Coll Cardiol 1994;24:755-62

14 Zabalgoitia M, Halperin JL, Pearce LA, et al. Transesophageal echocardiographic correlates of clinical risk of thromboembolism in nonvalvular atrial fibrillation. J Am Coll Cardiol 1998:31:1622-6.

15 Stroke Prevention in Atrial Fibrillation Investigators. Transesophageal echocardiographic correlates of thromboembolism in high risk patients with nonvalvular atrial fibrillation. The stroke prevention in atrial fibrillation investigators committee on echocardiography. Ann Intern Med 1998; 128:639-47.

16 Mügge A, Kühn $\mathrm{H}$, Nikutta $\mathrm{P}$, et al. Assessment of left atrial appendage function by biplane transesophageal echocardiography in patients with nonrheumatic atrial fibrillation: identification of a subgroup of patients at increased embolic risk. J Am Coll Cardiol 1994;23:599-607.

17 Henry WL, DeMaria A, Gramiak R, et al. Report of the American Society of Echocardiography committee on nomenclature and standards in two dimensional echocardiography. Circulation 1980;62:212-7.

18 Omran $\mathrm{H}$, Jung $\mathrm{W}$, Rabahieh $\mathrm{R}$, et al. Left atrial appendage function in patients with atrial flutter. Heart 1997;78:250-4

19 Seward JB, Khandheria BK, Freeman WK, et al. Multiplane transesophageal echocardiography: image orientation, examination technique, anatomic correlations and clinical applications. Mayo Clin Proc 1993;68:523-51.

20 Agmon Y, Khandheria BK, Gentile F, et al. Echocardiographic assessment of the left atrial appendage. J Am Coll Cardiol 1999;34:1867-77

21 Albers GW, Dalen JE, Laupacis A, et al. Antithrombotic therapy in atrial fibrillation. Chest 2001;119(suppl 1):194S-206S.

22 Brickner ME, Friedman DB, Cigarroa CG, et al. Relation of thrombus in the left atrial appendage by transesophageal echocardography to clinical risk factors for thrombus formation. Am J Cardiol 1994;74:391-3

23 Vigna C, Russo A, De Rito R, et al. Frequency of left atrial thrombi by ransesophageal echocardiography in idiopathic and ischemic dilated cardiomyopathy. Am J Cardiol 1992;70:1500-1.

\section{ELECTRONIC PAGES}

\section{eHEART: www.heartinl.com}

-he following electronic only articles are published in conjunction with this issue of Heart.

\section{Acute thrombosis of the sinus node artery: arrhythmological implications}

\section{G Ando', A Gaspardone, I Proietti}

A 53 year old woman was referred for percutaneous coronary intervention because of a recent inferior myocardial infarction. During right coronary artery stent implantation, intermittent occlusion of the coronary side branch for the sinus node occurred, associated with intermittent sinus arrest and junctional escape rhythm. This led to speculation about the potential mechanisms for sinus node dysfunction. Degenerative fibrosis of nodal tissue is actually considered the most common cause of bradyarrhythmias. Yet, in everyday practice, no particular attention is usually paid to other potential pathogenic mechanisms such as coronary artery disease. This may be particularly true for elderly patients or patients with multiple risk factors. Thus, sinus node dysfunction may be an unrecognised marker of coronary artery disease.

(Heart 2003;89:e5) www.heartjnl.com/cgi/content/full/89/2/e5
Intrastent sonotherapy in pulmonary vein restenosis: a new treatment for a recalcitrant problem

C J McMahon, C E Mullins, H G El Said

A 2 year old boy developed recurrent pulmonary vein stenosis after surgical repair of infradiaphragmatic pulmonary venous connection. He had required implantation of stents in the left and right sided pulmonary veins at 7 and 13 months of age, respectively. By 2 years of age he had undergone three catheterisation procedures and two surgical procedures to treat recurrent pulmonary vein stenosis. His right ventricular pressure was suprasystemic and catheterisation showed severe neointimal proliferation of both left and right sided stents. At this time the stents were dilated by balloon with simultaneous intrastent sonotherapy. Three months later the patient's clinical improvement was significant, his right ventricular pressure had decreased, and Doppler velocity had decreased across both left and right sided stents.

(Heart 2003;89:e6) www.heartjnl.com/cgi/content/full/89/2/e6 